\title{
É Seguro não Utilizar Sulfato de Magnésio nas Pacientes com Pré-Eclâmpsia?
}

Is it Safe to Withold Magnesium Sulfate in Preeclamptic Women?

José Geraldo Lopes Ramos, Sérgio Martins Costa, Mara Alexandra Francisco, Andrea Garcia de Almeida, Marta Franco Machado, Claudia Borowski

\section{RESUMO}

Objetivo: determinar a capacidade do sulfato de magnésio na prevenção da eclâmpsia em um grupo de gestantes com pré-eclâmpsia.

Métodos: estudo caso-controle em 489 gestantes com o diagnóstico de hipertensão arterial sistêmica (HAS) internadas no HCPA no periodo de janeiro de 1990 a janeiro de 1997. Para a aferição dos dados, as gestantes foram divididas em dois grupos: as que fizeram uso de sulfato de magnésio (Grupo I) e as que não fizeram uso do sulfato de magnésio (Grupo II). Todas as pacientes foram manejadas de acordo com o protocolo do Serviço de Ginecologia e Obstetricia do HCPA para tratamento de gestantes com HAS. Foram aferidas as seguintes variáveis: idade materna, raça, número de convulsões, número de convulsões em gestantes com uso de sulfato de magnésio, tempo de uso de sulfato de magnésio antes e após o parto, mortalidade materna; necessidade de internação em UTI, necessidade de ventilação assistida, e tempo de internação após o parto. Os dados foram avaliados por meio do pacote estatístico Epi-Info $6.0 \mathrm{com}$ análise multivariada. A principal medida foi o uso de sulfato de magnésio. Resultados: não foram encontradas diferenças significativas entre os grupos de usuárias ou não de sulfato de magnésio quanto à idade materna, idade gestacional ou raça. As gestantes que receberam $\mathrm{MgSO}_{4}$ apresentaram médias de pressões diastólica e sistólica significativamente maiores. O grupo de usuárias de sulfato de magnésio teve maior tempo de internação hospitalar e mais necessidade de internação em UTI. A necessidade de uso de respirador artificial $e$ o indice de morte materna foram semelhantes nos dois grupos. Vinte $e$ duas pacientes das 353 gestantes apresentaram uma ou mais convulsões antes da internação. Seis gestantes $(27,3 \%)$ apresentaram um ou dois novos episódios de convulsão e nenhuma paciente apresentou três ou mais convulsões após o uso sulfato de $\mathrm{MgSO}_{4}$.

Conclusões: os resultados do estudo sugerem que as convulsões eclâmpticas podem ser evitadas pela utilização profilática rotineira do sulfato de magnésio.Deve ser considerada uma medida arriscada e indefensável, sob o ponto de vista ético, a decisão de não-utilização profilática do sulfato de magnésio em pacientes pré-eclâmpticas que estejam em trabalho de parto, ou que apresentem sinais clinicos de iminência de convulsão.

PALAVRAS-CHAVE: Eclâmpsia. Hipertensão arterial. Mortalidade materna.

\section{Introdução}

A eclâmpsia continua sendo um problema grave nos países em desenvolvimento, constitu-

Departamento de Ginecologia e Obstetrícia, Faculdade de Medicina, Universidade Federal do Rio Grande do Sul

Correspondência:

Sérgio Martins Costa

Departamento de Ginecologia e Obstetrícia, Hospital de Clínicas de Porto Alegre (HCPA)

R. Ramiro Barcelos 2350 - S. 1125

90035-007 - Porto Alegre - RS

FAX: (51) 333-1681 indo umas das principais causas de mortalidade materna ${ }^{1}$. A utilização do sulfato de magnésio na prevenção e tratamento da eclâmpsia tem sido motivo de controvérsia ao longo dos anos ${ }^{2}$. Até 1995, a literatura médica não apresentava nenhum grande ensaio clínico randomizado que comprovasse a real eficácia e superioridade do sulfato de magnésio sobre outros anticonvulsivantes, tanto na profilaxia como no tratamento da eclâmpsia ${ }^{3}$. Vários centros de referência advogam o uso do sulfato de magnésio desde há muito tempo, apresentando excelentes indicadores de eficácia em 
séries de $\operatorname{casos}^{4,5}$. Em 1995, foram publicados os resultados de um grande ensaio clínico colaborativo sobre eclâmpsia, evidenciando a superioridade do magnésio sobre a fenitoína e o diazepam no tratamento da eclâmpsia ${ }^{6}$. Neste mesmo ano, Lucas et al. ${ }^{7}$ relataram outro estudo randomizado que comprovou a superioridade do sulfato de magnésio sobre a fenitoína na profilaxia da eclâmpsia em gestantes com hipertensão arterial. Neste estudo o critério de entrada para a randomização era o de apresentar valores da pressão arterial superiores a $140 / 90 \mathrm{mmHg}$, critério este considerado demasiadamente inespecífico por outros autores ${ }^{8}$.

O Serviço de Ginecologia e Obstetrícia do Hospital de Clínicas de Porto Alegre é um centro de referência para gestação de alto risco do sul do Brasil, que utiliza desde 1980 um protocolo de atendimento às gestantes com pré-eclâmpsia e eclâmpsia baseado no uso de sulfato de magnésio, conforme preconizado originalmente por Pritchard et al. ${ }^{5}$ no Parkland Memorial Hospital de Dallas (EUA). Neste protocolo, o sulfato de magnésio é prescrito para todas as gestantes com diagnóstico de pré-eclâmpsia (hipertensão arterial sistêmica [HAS] e proteinúria $\geq 300 \mathrm{mg} / 24 \mathrm{~h}$ ) que estejam em trabalho de parto ou que serão submetidas à cesariana, como, também, para todas as pacientes com pré-eclâmpsia que apresentem sinais premonitórios de convulsão, estando ou não em trabalho de parto, sendo o tratamento mantido nos casos mais graves por até 24 horas após o nascimento ${ }^{9}$.

O objetivo deste estudo é o de avaliar a eficácia do sulfato de magnésio na profilaxia das convulsões eclâmpticas com a hipótese operacional de que o mesmo tem eficácia preventiva.

\section{Pacientes e Métodos}

Foi realizado um estudo retrospectivo tipo caso-controle em gestantes com o diagnóstico de hipertensão arterial sistêmica. Os casos foram selecionados por meio do sistema de arquivamento médico do Serviço de Ginecologia e Obstetrícia do Hospital de Clinicas de Porto Alegre. Foram revisados 489 prontuários médicos de gestantes que haviam sido internadas na Maternidade do HCPA com o diagnóstico de hipertensão arterial sistêmica no período de janeiro de 1990 a janeiro de 1997. Foi considerada como hipertensão arterial sistêmica a ocorrência de duas medidas de pressão arterial de $140 / 90 \mathrm{mmHg}$ ou mais, efetuadas com um intervalo de 6 horas. A medida da pressão arterial era realizada com a paciente em posição sentada, com esfigmomanômetro do tipo aneróide aplicado no terço médio do braço, utilizando-se o $1^{\circ}$ e $5^{\circ}$ sons de Koroktov. Foram utilizados como critérios diagnósticos de hipertensão arterial os mesmos preconizados pelo Colégio Americano de Ginecologia e Obstetrícia. A hipertensão arterial era tratada quando os niveis eram superiores a 160/110 mmHg. A medicação empregada foi metildopa para a HAS crônica e hidralazina IV ou nifedipina VO para HAS aguda.

Para a aferição dos efeitos, as gestantes foram divididas em dois grupos: as que fizeram uso de sulfato de magnésio (casos-grupo I) e as que não fizeram uso do sulfato de magnésio (controles-grupo II). O sulfato de magnésio foi utilizado com dose de ataque endovenosa de 2 g logo que detectados os sinais premonitórios de eclâmpsia ou na eclâmpsia, adicionado da dose de ataque intramuscular de $5 \mathrm{~g}$ em cada nádega, seguida de uma dose de manutenção de $5 \mathrm{~g}$ por via intramuscular a cada 4 horas desde que estabelecidas as condições necessárias para a aplicação da droga: diurese acima de $25 \mathrm{ml} / \mathrm{h}$, presença de reflexos tendinosos profundos e freqüência respiratória acima de 12 movimentos respiratórios por minuto.

Os critérios de inclusão foram os seguintes: idade gestacional igual ou maior que 20 semanas; pressão arterial igual ou superior a 140/90 mmHg; assistência ao parto na Maternidade do HCPA. Os critérios de exclusão foram doença neurológica prévia associada (especialmente a epilepsia) e idade gestacional abaixo de 20 semanas. Foram aferidas as seguintes variáveis: idade materna, raça, número de convulsões, número de convulsões em gestantes com uso de sulfato de magnésio, tempo de uso de sulfato de magnésio antes e após o parto, mortalidade materna; necessidade de internação em Unidade de Tratamento Intensivo (UTI), necessidade de ventilação assistida e tempo de internação após o parto.

Os dados foram armazenados e avaliados através do pacote estatístico Epi-Info 6.0 com análise multivariada, sendo utilizado o teste do $\chi^{2}$ e o teste t de Student. Os dados foram coletados sob absoluto sigilo quanto à identificação de pacientes e equipe médica, o mesmo valendo para a divulgação dos resultados. O presente estudo foi aprovado pelo Comitê de Pesquisa e Ética do HCPA.

\section{Resultados}

Foram estudadas 489 gestantes com o diagnóstico de hipertensão arterial sistêmica que procuraram a Maternidade do Hospital de Clínicas de Porto Alegre no período de 1990 a 1997. Destas 
pacientes, 353 receberam o sulfato de magnésio durante o trabalho de parto como tratamento ou profilaxia de convulsões, ao passo que 136 não o utilizaram por apresentarem muito baixo risco para a convulsão (gestantes hipertensas sem proteinúria ou pré-eclâmpticas sem sinais premonitórios de convulsão e fora de trabalho de parto). A indicação do uso do sulfato de magnésio foi estabelecida pela equipe médica de plantão, não havendo interferência dos autores, mas obedecendo a rotinas do Serviço de Ginecologia e Obstetrícia do HCPA 9 .

Não foram encontradas diferenças significativas entre os grupos de usuárias ou não de sulfato de magnésio (Grupo I e Grupo II) quanto à idade materna, idade gestacional ou raça (Tabela 1 ). Contudo, o Grupo I apresentou uma média de pressões diastólica e sistólica significativamente mais elevadas, representando um grupo de gestantes com quadro clínico mais grave.

Tabela 1 - Características das gestantes com hipertensão arterial

\begin{tabular}{lccc}
\hline Variável & $\begin{array}{c}\text { Com MgSO }_{4} \\
(\mathbf{n}=353)\end{array}$ & $\begin{array}{c}\text { Sem MgSO } \\
(\mathbf{n}=136)\end{array}$ & p \\
\hline Raça branca & $284(80,4 \%)$ & $116(85,2 \%)$ & $\mathrm{NS}^{1}$ \\
Idade (anos) & $26,7 \pm 8,7$ & $27,6 \pm 10,2$ & $\mathrm{NS}^{2}$ \\
PAD (mmHg) & $108,0 \pm 20,3$ & $102,0 \pm 17,4$ & $0,002^{2}$ \\
PAS (mmHg) & $168,4 \pm 32,3$ & $159,5 \pm 31,0$ & $0,002^{2}$ \\
IG (semanas) & $34,5 \pm 6,5$ & $35,9 \pm 6,1$ & $\mathrm{NS}^{2}$ \\
\hline
\end{tabular}

NS $=$ não-significativo $\left({ }^{1}=\chi^{2} ;{ }^{2}=\right.$ teste $\mathrm{t}$ Student $)$

O tempo de internação do grupo de usuárias de sulfato de magnésio foi maior (média de 5 dias) com uma variação de 1 a 62 dias. A necessidade de utilização de Unidade de Tratamento Intensivo, de uso de respirador artificial e a taxa de morte materna foram semelhantes nos dois grupos (Tabela 2). Ocorreram 6 mortes maternas no grupo I $(1,7 \%)$ e 1 morte no grupo II $(0,7 \%)$.

Tabela 2 - Dias de internação, necessidade de UTI, uso de respirador e morte materna entre pacientes tratadas ou não com sulfato de magnésio.

\begin{tabular}{lccc}
\hline Variável & $\mathrm{Com} \mathrm{MgSO}_{4}$ & $\mathrm{Sem} \mathrm{MgSO}_{4}$ & $\mathbf{p}$ \\
\hline Internação (dias) & $5,0 \pm 5,9$ & $3,5 \pm 4,3$ & $0,00009^{2}$ \\
Variação & $1-62$ & $1-40$ & \\
Necessidade de UTI (n) & 17 & 2 & $\mathrm{NS}^{1}$ \\
Uso de respirador (n) & 4 & 1 & $\mathrm{NS}^{1}$
\end{tabular}

$\mathrm{NS}=$ não-significativo $\left({ }^{1}=\chi^{2} ;{ }^{2}=\right.$ teste $\mathrm{t}$ Student $)$
O tempo médio entre o uso do sulfato de magnésio e o parto foi de 10,2 horas, variando de 1 a 99 horas, com mediana de 5 horas. Após o parto, o sulfato de magnésio foi utilizado em média por até 17,5 horas, variando de 1 a 72 horas, com mediana de 21 horas.

Das 353 gestantes que fizeram uso do sulfato de magnésio $(4,5 \%$ do total das hipertensas e $6,2 \%$ das com HAS e proteinúria), 22 apresentaram eclâmpsia antes da internação, antes do uso de sulfato de magnésio. Dezessete pacientes apresentaram somente uma convulsão antes da internação e em um caso ocorreram 6 convulsões antes da internação. Estas pacientes configuraram o grupo de hipertensas que utilizaram o sulfato de magnésio como tratamento da eclâmpsia. Destas, 4 gestantes $(18,2 \%)$ apresentaram um novo episódio de convulsão e 2 um segundo episódio, com um índice geral de recorrência de $27,3 \%$. Nenhuma paciente apresentou a terceira convulsão após iniciar o sulfato de magnésio, não havendo necessidade de utilização de outro medicamento anticonvulsivante complementar.

Trezentos e trinta e uma pacientes foram tratadas com o sulfato de magnésio como profilaxia da eclâmpsia, seguindo os critérios acima referidos. Em 136 gestantes hipertensas, a equipe médica de plantão não identificou indicação para uso profilático de sulfato de magnésio. Em nenhuma destas 467 hipertensas ocorreu eclâmpsia.

No grupo total de 489 gestantes hipertensas, ocorreram 7 óbitos. Destes, 5 estavam associados ao sindrome HELLP, 4 à coagulação intravascular disseminada, 4 à eclâmpsia, 2 ao AVC e um à diabete melito (Tabela 3). Dos 4 casos de eclâmpsia seguida de morte, 2 ocorreram em pacientes cujo quadro convulsivo havia iniciado antes do tratamento com sulfato de magnésio e 2 em pacientes que receberam $\mathrm{MgSO}_{4}$ antes da primeira convulsão.

Tabela 3 - Fatores associados à mortalidade materna nas gestantes com hipertensão arterial sistêmica.

\begin{tabular}{lcc}
\hline & $\mathbf{n}$ & \% \\
\hline Síndrome de HELLP & 5 & 71,4 \\
Coagulação intravascular disseminada & 4 & 57,1 \\
Eclâmpsia & 4 & 57,1 \\
Acidente vascular cerebral & 2 & 28,6
\end{tabular}

Mortalidade materna: 7 de 489 casos (mais de um fator associado por morte materna). 


\section{Discussão}

O risco do surgimento da eclâmpsia e sua associação com aumento da morbidade e mortalidade materna e perinatal, principalmente nos países em desenvolvimento, tem justificado a larga utilização de terapia anticonvulsivante na prevenção de convulsões em gestantes com préeclâmpsia. Até a publicação dos resultados do The Eclampsia Collaborative Group ${ }^{6}$, o qual confirmou a superioridade do sulfato de magnésio sobre a fenitoína e o diazepam, a principal controvérsia entre os diferentes autores centrava-se na escolha da melhor droga anticonvulsivante para o tratamento das convulsões eclâmpticas.

Ultimamente permanece a dúvida sobre a validade ou não da utilização do sulfato de magnésio (ou outro anticonvulsivante) na profilaxia da eclâmpsia. Devido à baixa incidência da eclâmpsia, não existem bons ensaios clínicos prospectivos e randomizados com um número suficiente de casos com poder estatístico para responder esta dúvida. Burrows e Burrows ${ }^{10}$ relatam uma pequena incidência de convulsões em pacientes com pré-eclâmpsia ou pré-eclâmpsia sobreposta $(3,9 \%$ em 467 hipertensas). Esta baixa incidência justificaria um manejo sem a prevenção de convulsões, pois as mesmas não estariam associadas com o aumento de mortalidade materna ou perinatal. Da mesma forma, Brown e Buddle ${ }^{8}$ relataram um risco de convulsão de somente $0,6 \%$ em pacientes hipertensas com proteinúria mas sem sinais de eclâmpsia iminente, concluindo que há pequeno risco de eclâmpsia se a profilaxia das convulsões não for feita.

No nosso estudo, encontramos uma incidência de $4,5 \%$ de eclâmpsia no grupo completo de hipertensas, sendo que no grupo em que foi realizada a profilaxia "primária" de eclâmpsia não ocorreu uma única convulsão. O percentual de convulsões esperado para este grupo de pacientes, segundo o estudo de Burrows e Burrows ${ }^{10}$, seria de no mínimo 3,9\%. Se considerarmos uma incidência de 3,9\% de eclâmpsias, seriam esperados pelo menos 13 novos casos de eclâmpsia nas 353 pacientes tratadas com sulfato de magnésio profilaticamente, vários dos quais poderiam se associar ao desfecho de morte materna. Portanto, acreditamos que este risco provável de quase $4 \%$ de ocorrência de convulsões justifica plenamente a utilização de medidas com o potencial de ação preventiva como o apresentado pelo sulfato de magnésio. Além disso, o fato de o grupo que utilizou sulfato de magnésio ter apresentado um tempo maior de internação, uma maior necessidade de tratamento em UTI e medidas mais elevadas de pressão arterial reflete um grupo de pacientes mais graves e portanto mais propensas a apresentarem convulsões.

Este estudo de casos feito por um período de sete anos em um centro de referência para gestantes de alto risco, avaliando a utilização do sulfato de magnésio em mulheres grávidas com préeclâmpsia e eclâmpsia, sugere que as convulsões eclâmpticas podem ser evitadas pela utilização profilática rotineira do sulfato de magnésio. Portanto, no nosso hospital, até que se obtenham melhores conhecimentos sobre a patogênese da eclâmpsia e que surjam tratamentos mais efetivos, deve ser considerada uma medida arriscada e indefensável, sob o ponto de vista ético, a decisão de não-utilização profilática do sulfato de magnésio em pacientes pré-eclâmpticas que estejam em trabalho de parto, ou que apresentem sinais clínicos de iminência de convulsão.

\section{SUMMARY}

Purpose: to determine magnesium sulfate effectiveness to prevent eclamptic seizures in pregnant women diagnosed with preeclampsia.

Methods: a case-control study involving 489 pregnant patients admitted to the Hospital de Clinicas de Porto Alegre (HCPA) with the diagnosis of hypertension between January 1990 and January 1997. The patients were divided into two groups: those who received magnesium sulfate (Group I) and those who did not (Group II). All patients were treated according to the hospital's standard protocol for the treatment of hypertensive pregnant women. The following variables were assessed: maternal age, race, number of seizures, number of seizures in patients receiving magnesium sulfate, period of magnesium sulfate administration, before and after delivery, maternal mortality, need of ICU care, need of mechanical ventilation and length of hospital stay after delivery. Data were assessed with Epi-Info 6.0 statistical package, using multivariate analysis. The main outcome measure was magnesium sulfate use.

Results: no differences were found between the two groups in terms of maternal age, race or gestational age. Women who received magnesium sulfate had a higher mean systolic and diastolic blood pressure. Women in the intervention group had a longer hospital stay and a greater need of ICU care. Need of mechanical ventilation and maternal mortality were similar in the two groups. Twenty-two out of 353 women had one or more seizures before admission to the hospital. Six women (27.3\%) had one or two additional seizures after magnesium sulfate administration. No subjects had three or more seizures after receiving magnesium sulfate.

Conclusion: the results show that eclamptic seizures can be prevented by routine prophylactic magnesium sulfate administration.

KEY WORDS: Eclampsia. Hypertension. Maternal mortality. 


\section{Referências}

1.Engelhardt T, Moodley J, Mothabani B. Does antenatal care in developing countries prevent eclampsia? A retrospective analysis at King Edward VIII Hospital, Durban, South Africa. Hypertens Pregnancy 1996; 15:87-94.

2.Donaldson JO. The case against magnesium sulphate for eclamptic convulsions. Int $\mathrm{J}$ Gynaecol Obstet 1992; 337:250-1.

3. Moodley J, Moodley VV. Prophylactic anticonvulsivant therapy in hypertensive crisis of pregnancy - The need of a large, randomized trial. Hypertens Pregnancy 1994; 13:245-52.

4.Zuspan FP. Problems encountered in the treatment of pregnancy-induced hypertension. A point of view. Am J Obstet Gynecol 1978; 131:591-7.

5.Pritchard JA, Cunningham FG, Pritchard SA. The Parkland Memorial Hospital protocol for treatment of eclampsia: evaluation of 245 cases. Am J Obstet Gynecol 1984; 148:951-63.
6.Duley L, Carroli G, Belizan J. Which anticonvulsivant for women with eclampsia - evidence from the eclampsia trial. Lancet 1995; 345:1455-63.

7.Lucas MJ, Leveno KJ, Cunningham FG. A comparison of magnesium-sulfate with phenytoin for the prevention of eclampsia. N Engl J Med 1995; 333:201-5.

8.Brown MA, Buddle ML. Is it safe to withhold convulsion prophylaxis in preeclamptic women without neurological features? Hypertens Pregnancy 1998; 17:13-22.

9.Martins-Costa S, Ramos JG, Barros E. Doença hipertensiva na gravidez. In: Freitas FM, Martins-Costa S, Ramos JG, Magalhães JA, editores. Rotinas em Obstetrícia. $3^{\mathrm{a}}$ ed. Porto Alegre: Artes Médicas; 1997. p. 272-85.

10.Burrows RF, Burrows EA. The feasibility of a control population for a randomized control trial of seizure prophylaxis in the hypertensive disorders of pregnancy. Am J Obstet Gynecol 1995; 173:929-35. 